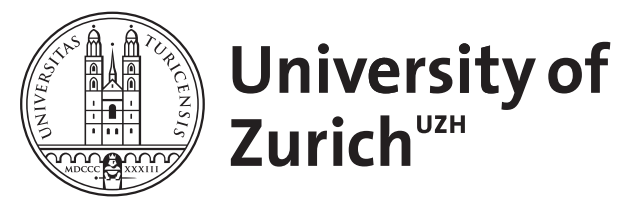

\title{
Retromers in Alzheimer's disease
}

Siegenthaler, Barbara ; Rajendran, Lawrence

\begin{abstract}
Amyloid- peptide (A ), the key pathogenic agent in Alzheimer's disease (AD), is released after sequential proteolytic cleavage of the transmembrane amyloid precursor protein (APP). -Site APPcleaving enzyme 1 (BACE1) cleaves APP in early endosomes, and the cause of increased BACE cleavage of APP in AD is not fully resolved yet. It has been proposed that perturbed intracellular trafficking of APP, which leads to prolonged residence time in early endosomes, influences A production and hence the risk for $\mathrm{AD}$. Retromers are a family of proteins that mediate the retrieval of transmembrane proteins from the endosomes to the trans-Golgi network. Misregulation of retromers or retromer-associated proteins influences endosomal localization of APP/BACE1. Here we review the role of retromers in the amyloidogenic processing of APP and their pathogenic role in AD.
\end{abstract}

DOI: https://doi.org/10.1159/000335910

Posted at the Zurich Open Repository and Archive, University of Zurich ZORA URL: https://doi.org/10.5167/uzh-63011

Journal Article

Published Version

Originally published at:

Siegenthaler, Barbara; Rajendran, Lawrence (2012). Retromers in Alzheimer's disease. Neurodegenerative Diseases, 10(1-4):116-121.

DOI: https://doi.org/10.1159/000335910 
Neurodegenerative Dis 2012;10:116-121

DOI: $\underline{10.1159 / 000335910}$
Received: August 3, 2011

Accepted after revision: December 18, 2011

Published online: March 3, 2012

\title{
Retromers in Alzheimer's Disease
}

\author{
Barbara M. Siegenthaler Lawrence Rajendran \\ Systems and Cell Biology of Neurodegeneration, Division of Psychiatry Research, University of Zurich, \\ Zurich, Switzerland
}

\section{Key Words}

Retromers $\cdot$ Alzheimer's disease $\cdot$ Amyloid- $\beta$ peptide

\begin{abstract}
Amyloid- $\beta$ peptide $(A \beta)$, the key pathogenic agent in Alzheimer's disease (AD), is released after sequential proteolytic cleavage of the transmembrane amyloid precursor protein (APP). $\beta$-Site APP-cleaving enzyme 1 (BACE1) cleaves APP in early endosomes, and the cause of increased BACE cleavage of APP in AD is not fully resolved yet. It has been proposed that perturbed intracellular trafficking of APP, which leads to prolonged residence time in early endosomes, influences $A \beta$ production and hence the risk for AD. Retromers are a family of proteins that mediate the retrieval of transmembrane proteins from the endosomes to the trans-Golgi network. Misregulation of retromers or retromer-associated proteins influences endosomal localization of APP/BACE1. Here we review the role of retromers in the amyloidogenic processing of APP and their pathogenic role in AD.
\end{abstract}

Copyright $\odot 2012$ S. Karger AG, Basel

\section{Introduction}

Alzheimer's disease (AD), the most common form of dementia, is a genetically complex neurological disorder with excessive neurodegeneration resulting in the progressive loss of memory function and cognitive abilities
[1]. Two characteristic pathological changes are observed in AD patient brains: intracellular tangles and extracellular plaques in the hippocampus, cerebral cortex and in other areas of essential function for cognitive abilities [2]. Plaques, which are thought to be a cause of AD, consist of secreted, aggregated and accumulated $\beta$-amyloid peptides $(A \beta)$ [3]. $A \beta$ is a cleavage product that is released after proteolytic processing of a transmembrane protein called the amyloid precursor protein (APP). APP plays a central role in the pathogenesis of $\mathrm{AD}$ and, depending on its intracellular trafficking, it is processed either by $\alpha$ secretase to the nonamyloidogenic soluble fragment sAPP $\alpha$ or by $\beta$-secretase to sAPP $\beta$ and a C-terminal fragment that is further processed to the amyloidogenic $\mathrm{A} \beta[2]$.

APP, $\beta$-site APP-cleaving enzyme 1 (BACE1) and the $\gamma$-secretase complex are all integral membrane protein components and hence are subjected to the regulation by intracellular trafficking. Recent evidence points out to the role of endocytosis by which APP and BACE1 are internalized into early endosomes where $A \beta$ production occurs [4]. Proteins that modulate the endosomal residency time of these proteins thus could modulate the production of $A \beta$ and hence have implications for AD. One group of such proteins are retromers that mediate the retrieval of transmembrane proteins from endosomes to the trans-Golgi network (TGN) [5]. Retromers are heteropentameric proteins which bind to the cytosolic side of endosomes and mediate the retrograde transport of

\section{KARGER}

Fax +4161306 1234

E-Mail karger@karger.ch

www.karger.com
(C) 2012 S. Karger AG, Basel

$1660-2854 / 12 / 0104-0116 \$ 38.00 / 0$

Accessible online at:

www.karger.com/ndd
Lawrence Rajendran

Systems and Cell Biology of Neurodegeneration

Division of Psychiatry Research, University of Zurich

August-Forel Strasse 1, CH-8008 Zurich (Switzerland)

Tel. +41 4463488 71, E-Mail rajendran@bli.uzh.ch 
transmembrane proteins to the Golgi. Loss of retromer function has been correlated with risk for AD and elevated levels of $A \beta$ peptide. In this review, we outline the basic aspects of retromer structure and function and its involvement in $\mathrm{AD}$.

\section{Cell Biology of Amyloid Production}

The amyloidogenic peptide, $A \beta$, that is causally linked to $\mathrm{AD}$, is liberated from the APP via proteolytic processing by $\beta$ - and $\gamma$-secretases. A transmembrane aspartyl protease, also termed BACE1, confers $\beta$-secretase activity by cleaving APP to generate A $\beta$ [3]. $\gamma$-Secretase is a multicomponent complex that is composed of presenilin-1/ presenilin-2, Aph-1 (in humans, Aph-1a or Aph-1b), nicastrin, and Pen-2 $[6,7]$. APP and $\beta$-secretase are synthesized in the endoplasmic reticulum, glycosylated to mature proteins and then transported to the plasma membrane from where they are endocytosed into endosomes.

$\beta$-Secretase cleavage of APP occurs predominantly in endosomes [4, 8-12]. The $\mathrm{pH}$ of endosomes ( $\mathrm{pH}$ 4.5-6.0) is optimal for $\beta$-secretase activity, which explains the requirement for endocytosis. On the other hand, $\alpha$-secretase cleavage of APP, which precludes the production of the toxic $A \beta$ peptide, occurs at the plasma membrane [13]. $\gamma$-Secretase components are also synthesized in the endoplasmic reticulum but the assembly of the mature and functional complex requires the coordinated regulation of the endoplasmic reticulum-Golgi recycling circuit $[14,15]$ and APP cleavage by $\gamma$-secretase, to generate $A \beta$, occurs in post-Golgi compartments, i.e. in endosomes [16].

$A \beta$ that is produced in endosomes can be sorted to retrograde compartments such as multivesicular bodies via vesicular trafficking. Release of $A \beta$ from the intracellular compartments is shown to occur via the fusion of multivesicular bodies with the plasma membrane $[10,17]$. This fusion releases the intraluminal vesicles of multivesicular bodies as exosomes, and we have shown that a fraction of $A \beta$ is released in association with exosomes [10]. Although the pathway by which the majority of $A \beta$ is released is still unknown, various pieces of evidence suggest that the multivesicular body fusion pathway could also release the bulk of $A \beta$ which could contribute to the formation of amyloid plaques [18-20].

Since $A \beta$ production occurs in endosomes, mechanisms that regulate the trafficking of the substrate APP and its secretases ( $\alpha$-, $\beta$ and $\gamma$-secretases) critically determine the levels of $A \beta$ produced. Several observations support the involvement of endocytosis in $\beta$-amyloid generation [9, 21-25]. For example inhibition of dynamin, a GTPase that pinches off endocytic vesicles during internalization, dramatically reduces $\beta$-cleavage of APP and also $A \beta$ production $[10,11,26]$. Mutating the cytosolic sorting signals of APP or BACE1, targeting a BACE1 inhibitor to endosomes [27] or overexpression of rab4, a small GTPase that mediates the immediate recycling of cargo from early endosomes [10], all decreased the generation of $A \beta$. These results conclusively showed that the amyloidogenic processing of APP occurs in endosomes. Hence retrieval or removal of APP or BACE1 from endosomal compartments should lead to a decreased production of $A \beta$. Many papers suggest that retromers and retromer-associated proteins could regulate $A \beta$ production [28-34]. In accordance with these observations, mutations/polymorphisms leading to retromer dysfunction have been suggested to be a risk factor of AD.

\section{Retromers}

First identified in yeast [35], retromers were reported as membrane coat complex proteins that mediate the retrieval of the acid hydrolase receptors from endosomes (vacuole in yeast) to the Golgi apparatus. Acid hydrolases are sorted to endosomes with the help of the sorting receptors such as the yeast vacuolar protein sorting 10 (VPS10) or mammalian mannose-6-phosphate receptors. Once the cargo (in this case, acid hydolases) is delivered to endosomes, the receptors have to be shuttled back to the Golgi in order to initiate a new round of transport to endosomes. This retrieval from the endosomes back to the TGN is carried out by retromers. Retromers are heteropentameric protein complexes composed of mainly two components: a structural part made of a dimer of sorting nexins (SNX), and he cargo recognition complex composed of a vacuolar protein sorting trimer [36-38].

\section{Sorting Nexins}

The sorting nexins contain a phox homology (PX) domain that mediates the binding to phosphoinositides in the endosomal membrane. In that way they are able to recruit the interacting VPS retromer subunits to endosomes. In mammals, the SNX dimer is mainly formed by either SNX1/SNX2 and SNX5/SNX6. In addition to the PX domain, SNX1 and 2 also contain a BAR (Bin, Amphiphysin and Rvs) domain that senses curvature in membranes and aids in membrane tubulation, a process critically important for the transport of proteins from en- 
dosomes $[39,40]$. A recent study showed that SNX3, not the canonical SNX1-2 or 5-6, mediates the retrograde sorting of Wnt receptor, Wntless. This study showed that SNX3 constitutes an alternative retromer pathway and is involved in the secretion of Wnt [41].

\section{Cargo Recognition Complex}

In retromers, the binding to the cargo (the transmembrane protein that needs to be retrieved) is mediated by the VPS26-VPS29-VPS35 complex. VPS35 builds the core element of the retromer complex and binds to VPS26 (two isoforms VPS26A and VPS26B), VPS29 and SNX proteins [37, 42]. The role of the two isoforms of VPS26 is not yet clear. Structural studies show that both VPS29 and VPS26 can bind independently to VPS35. Since SNX can sense/induce curvature, retromers have been proposed to serve as 'coats' for tubulovesicular carriers. Consistent with this assumption, retromer proteins have been shown to colocalize with markers of early endosomes, late endosomes and some tubular endocytic vesicles that carry the cargo to the TGN [35-38]. Cells deficient in VPS26 (either derived from knockout mice or RNAi silenced) fail to retrieve the acid hydrolase receptor protein (cation-independent mannose-6-phosphate receptor) from endosomes thereby leading to either recycling the protein to the plasma membrane or sorting it for lysosomal degradation [43]. Hence retromers could be viewed as part of a sorting machinery that reroutes transmembrane proteins from lysosomal degradation to recycling via TGN.

\section{Retromers in APP Processing and AD}

\section{APP Trafficking by Retromers}

In 2005, three independent landmark studies from the labs of Scott Small, Jordan Tang and Thomas Willnow showed a possible involvement of retromers in $\mathrm{AD}[28$, $32,33]$.

Using model-guided microarray technique to study the spatiotemporal control of AD-related genes, Small et al. [33] first discovered that the retromer component, VPS35 mRNA levels were misregulated in the brain region most vulnerable in $\mathrm{AD}$. In addition, protein levels of VPS26 were also slightly reduced in the entorhinal cortex of AD patient samples. While the protein levels of VPS35 were low in AD patients, the mRNA levels were considerably higher. This inverse relationship of high mRNA and low protein could be attributed to many reasons including accelerated degradation of the VPS35 protein and slower turnover of its mRNA. Experimentally induced reduction of VPS35 using siRNA in HeLa cells increased A 340 levels by $37 \%$ and overexpression of VPS35 decreased $A \beta 40$ levels by $40 \%$. These results suggest that retrieval of APP from endosomes to TGN regulates A $\beta$ production $[4,5]$. Recently, it was shown that this retrieval is dependent on APP phosphorylation at its serine 655 residue [44]. In an attempt to establish a functional link between the retromer complex and $A \beta$-dependent behavioral deficits observed in late-onset AD (LOAD), Small and his group also characterized two genetically modified animal models addressing the role of retromers in $\mathrm{AD}$ [34]. They observed altered hippocampal-dependent memory function and long-term potentiation in the retromer-deficient mice and an increase in neuronal loss in retromer-deficient flies which expressed human APP and BACE1. This neuronal loss was accompanied by accumulation of human $A \beta$ peptides, suggesting a functional correlation between retromers and $A \beta$-induced neurodegeneration.

A few years ago, Thomas Willnow and his group identified a sorting protein-related receptor containing lowdensity lipoprotein receptor class A repeats (SorLA), a transmembrane protein that is expressed in neurons to be involved in the intracellular transport and processing of APP to A $\beta$ [28]. Sorting protein-related receptor (SorLA, encoded by the SORL1 gene) is a type 1 membrane protein with structural similarity to VPS10. Through this sequence, SorLA binds to the retromer complex and serves as an adaptor protein $[29,31]$ and serves as an adaptor protein for interacting proteins such as APP. Retromers can bind to APP in endosomal compartments and transport it retrogradely to the TGN. It has been shown that SorLA levels are reduced in affected brain areas of AD patients [28]. Furthermore, perturbations within this retrograde transport are shown to be associated with increased production of $A \beta$, neurodegeneration and finally LOAD [28]. But the exact mechanism of retromer function in APP trafficking and $A \beta$ secretion is still unknown. Willnow and colleagues found that SorLA interacted with APP and that both proteins colocalized in endosomal and Golgi compartments. Moreover, overexpression of SorLA redistributed APP to Golgi thereby reducing the residency time of APP and BACE in the early endosomes (fig. 1). Consequently, $A \beta$ levels were decreased in SorLA overexpression conditions while SorLA ablation in knockout mice increased $A \beta$ levels. Interestingly, frontal cortex samples from $\mathrm{AD}$ patients showed decreased SorLA expression compared to healthy controls. 


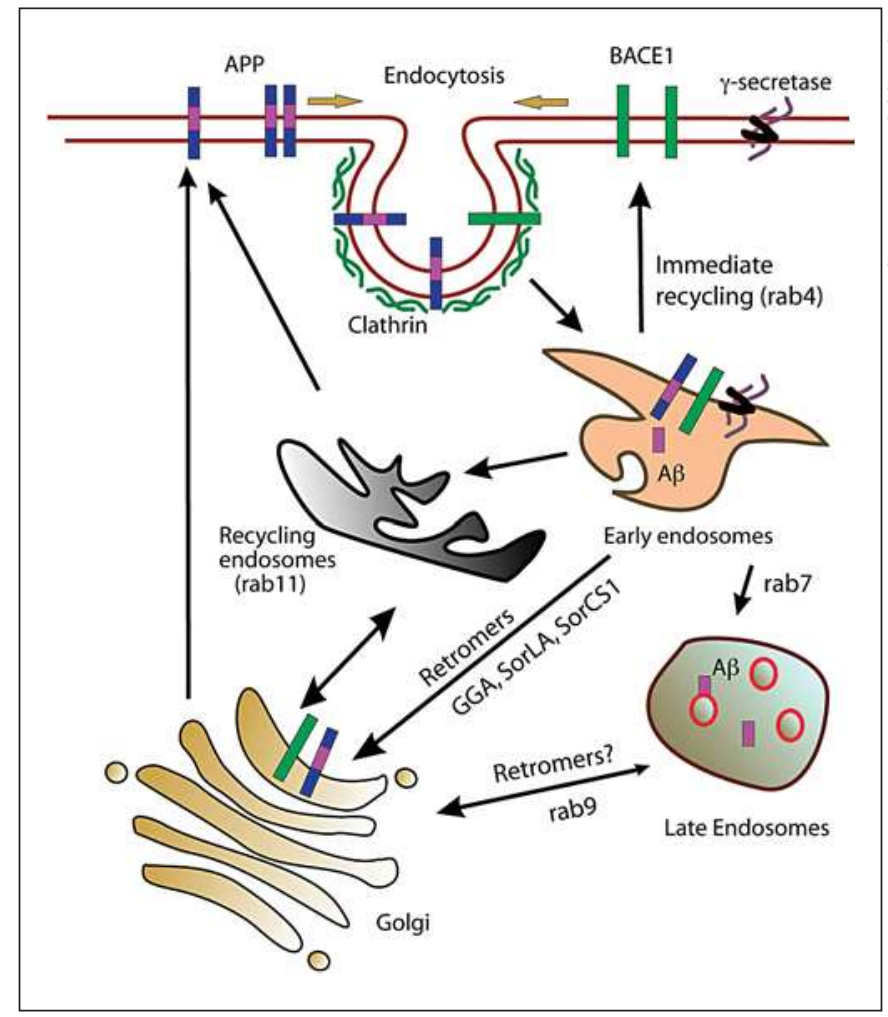

Fig. 1. Retromer-mediated sorting at the endosomes. APP, BACE1 and $\gamma$-secretase complex are trafficked to the cell surface and internalized into early endosomes. In the early endosomes, the $\mathrm{pH}$ is conducive for $\beta$-secretase cleavage of APP. APP retrieval from the early endosomes could occur via a rab4-mediated sorting directly to the plasma membrane. Alternatively, retromer proteins bind to retrieval receptors such as SorLA/SorCS1 which in turn bind to APP and mediate their transport to the TGN. In the absence of functional retromers or retrieval receptors, APP (or BACE1 in the case of GGA3) localization in endocytic compartments is enhanced leading to enhanced production of $A \beta$.

The relevance of these findings to $\mathrm{AD}$ was reinforced when Peter St. George-Hyslop's group [30] identified inherited variants of SORL1 that were associated with LOAD. Since retromer proteins have been shown to regulate $A \beta$ generation in endosomes and because they were misregulated in $\mathrm{AD}$, Rogaeva et al. [30] investigated the genetic association between $\mathrm{AD}$ and singlenucleotide polymorphisms in VPS10P-containing proteins, sortilin and sortilin-related receptors. They found 6 single-nucleotide polymorphisms in SORL1 that are significantly associated with AD. Out of these six single-nucleotide polymorphisms, three of them have been shown to decrease SORL1 expression by approximately $50 \%$ in haplotype carriers. TIn this study, it was also shown endogenous SORL1 directly interacted with the endogenous APP holoprotein and with VPS35 but does not interact with BACE1. While overexpression of SORL1 decreased A $\beta$ production, RNAi-mediated silencing of SORL1 increased SAPP $\beta$ (149\%) and A $\beta$ production (202\%) in this study. Interestingly, VPS26A knockdown significantly increased $A \beta 40$ (186\%) and A $\beta 42$ (183\%). Similar results were obtained in fly and mouse models of AD. These studies clearly demonstrated that SORL1 plays a key physiological role in the differential sorting of APP holoprotein and that retromer deficiency contributes to the pathogenesis of AD.

Several other studies have substantiated the retromer findings. Recently another retromer-associated protein, SorCS1, was also found to be involved in APP metabolism [45]. Here again, overexpression of SorCS1 reduced $A \beta$ generation. SorCS1, also implicated in type 1 and 2 diabetes mellitus, interacts with APP, SORL1 and VPS35. Keeping in line with these observations, SorCS1 hypomorphic mice exhibited a profound decrease in VPS35 levels (49\%). SorCS1 hypomorphic mice had increased levels of $\alpha / \beta$ $\mathrm{CTF}, A \beta 40$ and $A \beta 42$. Interestingly, these differences were found when hypomorphic female mice were compared to wild-type female mice, but not when males and females were compared as a group. Interestingly, SorCS1 is a VPS10 family member and all these results suggest that SorCS1 might have the same role as SORL1 in APP metabolism. One other study also showed that SorCS1 negatively regulated $A \beta$ production and found that inherited variants in SorCS1 were associated with AD [46].

While all these studies showed that retromers negatively regulate amyloidogenic APP processing one recent study, however, found that retromer disruption decreased $A \beta 40$ secretion such that the $A \beta 42 / A \beta 40$ ratio was changed [47]. They found that RNAi-mediated silencing of VPS35, the core component of the retromer complex, changed neither the amount nor the cellular distribution of total cellular APP. They also found no effect on the endocytosis of cell surface APP. However, VPS35 knockdown increased the ratio of secreted $A \beta 42 / A \beta 40$ mostly due to a decrease in $A \beta 40$ secretion. The authors found that retromer disruption upon VPS35 knockdown significantly increased secretion of APP C-terminal fragments in exosomal vesicles thereby reducing the amount of $A \beta$ production. How do these results fit with the previous observations that retromer disruption indeed increases $\beta$-cleavage of APP and A $\beta$ production? While this study concentrated on VPS35, it could still be possible that other components have retromer-complex-independent functions. 
Retromer-Dependent Trafficking of BACE1

Not only APP but also BACE1 has been shown to be dependent on retromer-mediated trafficking. As early as 2005, Jordan Tang's group [32] showed that BACE1 trafficking from endosomes to TGN is dependent on a protein called GGA (Golgi-localized $\gamma$-ear-containing ARFbinding protein) which facilitates the recycling of BACE1 (fig. 1). This study was also one of the first to show that a retromer subunit, VPS26, is directly involved in APP processing. In line with this observation, caspase-mediated cleavage of GGA-3 during apoptosis has been shown to stabilize $\mathrm{BACE} 1$ and enhance $\mathrm{A} \beta$ production [48]. Another retromer-associated protein, sortilin, is shown to bind BACE1 and a C-terminal truncated sortilin redistributed BACE1 to endosomes [49]. This study showed that overexpression of sortilin increased $\beta$-cleavage of $A P P$ and A $\beta$ levels whereas its reduction via RNAi decreased the amyloidogenic processing of APP. These findings, however, are in sharp contrast with the previously reported role of retromers in APP processing as this study shows a positive role for retromers in $A \beta$ production. The reason for this is unclear but reduced recycling of BACE1 in the absence of functional sortilin levels is attributed for the reduction in $A \beta$ production. In support of this, the amount of sortilin has been shown to be elevated in $\mathrm{AD}$ patients [48]. Recently, in a proteomic characterization, SNX6 was identified as a BACE1-interacting protein and validation experiments suggest that this SNX6-dependent trafficking of BACE1 regulates $A \beta$ production [50].

\section{Conclusion and Outlook}

The findings on retromers and APP processing further underscore the importance of membrane trafficking in $\mathrm{AD}[51,52]$. However, a lot more questions need to be addressed. Where do retromers act in the endocytic compartment? Since they regulated $A \beta$ production which largely occurs in early endosomes, it is suggested that the retromer pathway could act at the level of early endosomes. It is not yet clear whether retromer-associated proteins act at the level of early or late endosomes or both. A recent study on the intracellular transport of Furin and TGN38, two proteins that recycle between the TGN and plasma membrane, suggests that while TGN38 trafficking is retromer-dependent, trafficking of Furin involved rab9, a rab protein involved in late endosome-TGN [53]. Through extension, this study suggests that retromers probably act at the level of early endosomes and that cargoes that are retrieved from the late endosomal compartments are retromer-independent.

Are $\gamma$-secretase components retrieved by retromers from the endosomes? How do the polymorphisms in SORL1 and SorCS1 affect the retrieval of Wntless and thereby Wnt signaling [54]? Disrupted Wnt signaling is observed in AD [55]. It would be interesting to study if there is an underlying common cause through retromers. How are APP mutations affected through this trafficking pathway? Is Notch trafficking affected through retromers? What are the roles of retromers in synaptic function and dysfunction? Clearly an exciting period lies ahead to understand the molecular and cellular basis of LOAD.

\section{References}

1 Forman MS, Trojanowski JQ, Lee VM: Neurodegenerative diseases: a decade of discoveries paves the way for therapeutic breakthroughs. Nat Med 2004;10:1055-1063.

2 De Strooper B: Proteases and proteolysis in Alzheimer disease: a multifactorial view on the disease process. Physiol Rev 2010;90:465-494.

3 Haass C, Selkoe DJ: Soluble protein oligomers in neurodegeneration: lessons from the Alzheimer's amyloid beta-peptide. Nat Rev Mol Cell Biol 2007;8:101-112.

4 Small SA, Gandy S: Sorting through the cell biology of Alzheimer's disease: intracellular pathways to pathogenesis. Neuron 2006;52: 15-31.

5 Small SA: Retromer sorting: a pathogenic pathway in late-onset Alzheimer disease. Arch Neurol 2008;65:323-328.
6 De Strooper B: Aph-1, Pen-2, and Nicastrin with Presenilin generate an active gammaSecretase complex. Neuron 2003;38:9-12.

7 Vetrivel KS, Cheng H, Lin W, Sakurai T, Li T, Nukina N, et al: Association of gammasecretase with lipid rafts in post-Golgi and endosome membranes. J Biol Chem 2004; 279:44945-44954.

8 Koo EH: The beta-amyloid precursor protein (APP) and Alzheimer's disease: does the tail wag the dog? Traffic 2002;3:763-770.

9 Kinoshita A, Fukumoto H, Shah T, Whelan CM, Irizarry MC, Hyman BT: Demonstration by FRET of BACE interaction with the amyloid precursor protein at the cell surface and in early endosomes. J Cell Sci 2003;116: 3339-3346.
10 Rajendran L, Honsho M, Zahn TR, Keller P, Geiger KD, Verkade P, et al: Alzheimer's disease beta-amyloid peptides are released in association with exosomes. Proc Natl Acad Sci USA 2006;103:11172-11177.

11 Ehehalt R, Keller P, Haass C, Thiele C, Simons K: Amyloidogenic processing of the Alzheimer beta-amyloid precursor protein depends on lipid rafts. J Cell Biol 2003;160: 113-123.

12 He X, Cooley K, Chung CH, Dashti N, Tang J: Apolipoprotein receptor 2 and X11 alpha/ beta mediate apolipoprotein E-induced endocytosis of amyloid-beta precursor protein and beta-secretase, leading to amyloid-beta production. J Neurosci 2007;27:4052-4060. 
13 Kojro E, Gimpl G, Lammich S, Marz W, Fahrenholz F: Low cholesterol stimulates the nonamyloidogenic pathway by its effect on the alpha-secretase ADAM 10. Proc Natl Acad Sci USA 2001;98:5815-5820.

14 Spasic D, Annaert W: Building gammasecretase: the bits and pieces. J Cell Sci 2008; 121:413-420.

15 Spasic D, Raemaekers T, Dillen K, Declerck I, Baert V, Serneels L, et al: Rerlp competes with APH-1 for binding to nicastrin and regulates gamma-secretase complex assembly in the early secretory pathway. J Cell Biol 2007;176:629-640.

16 Kaether C, Schmitt S, Willem M, Haass C: Amyloid precursor protein and notch intracellular domains are generated after transport of their precursors to the cell surface. Traffic 2006;7:408-415.

17 Aguzzi A, Rajendran L: The transcellular spread of cy tosolic amyloids, prions, and prionoids. Neuron 2009;64:783-790.

18 Takahashi RH, Almeida CG, Kearney PF, Yu F, Lin MT, Milner TA, et al: Oligomerization of Alzheimer's beta-amyloid within processes and synapses of cultured neurons and brain. J Neurosci 2004;24:3592-3599.

19 Rajendran L, Knobloch M, Geiger KD, Dienel S, Nitsch R, Simons K, et al: Increased Abeta production leads to intracellular accumulation of Abeta in flotillin-1-positive endosomes. Neurodegener Dis 2007;4:164170 .

20 Gouras GK, Almeida CG, Takahashi RH: Intraneuronal Abeta accumulation and origin of plaques in Alzheimer's disease. Neurobiol Aging 2005;26:1235-1244.

21 Cataldo A, Rebeck GW, Ghetri B, Hulette C, Lippa C, Van Broeckhoven C, et al: Endocytic disturbances distinguish among subtypes of Alzheimer's disease and related disorders. Ann Neurol 2001;50:661-665.

22 Cataldo AM, Barnett JL, Pieroni C, Nixon RA: Increased neuronal endocytosis and protease delivery to early endosomes in sporadic Alzheimer's disease: neuropathologic evidence for a mechanism of increased betaamyloidogenesis. J Neurosci 1997; 17:61426151.

23 Cirrito JR, Kang JE, Lee J, Stewart FR, Verges DK, Silverio LM, et al: Endocytosis is required for synaptic activity-dependent release of amyloid-beta in vivo. Neuron 2008; 58:42-51.

24 Grbovic OM, Mathews PM, Jiang Y, Schmidt SD, Dinakar R, Summers-Terio NB, et al: Rab5-stimulated up-regulation of the endocytic pathway increases intracellular betacleaved amyloid precursor protein carboxylterminal fragment levels and Abeta production. J Biol Chem 2003;278:31261-31268.

25 Goodger ZV, Rajendran L, Trutzel A, Kohli BM, Nitsch RM, Konietzko U: Nuclear signaling by the APP intracellular domain occurs predominantly through the amyloidogenic processing pathway. J Cell Sci 2009; 122:3703-3714
26 Carey RM, Balcz BA, Lopez-Coviella I, Slack BE: Inhibition of dynamin-dependent endocytosis increases shedding of the amyloid precursor protein ectodomain and reduces generation of amyloid beta protein. BMC Cell Biol 2005;6:30.

27 Rajendran L, Schneider A, Schlechtingen G, Weidlich S, Ries J, Braxmeier T, et al: Efficient inhibition of the Alzheimer's disease beta-secretase by membrane targeting. Science 2008;320:520-523.

28 Andersen OM, Reiche J, Schmidt V, Gotthardt M, Spoelgen R, Behlke J, et al: Neuronal sorting protein-related receptor sorLA/ LR11 regulates processing of the amyloid precursor protein. Proc Natl Acad Sci USA 2005;102:13461-13466.

29 Andersen OM, Schmidt V, Spoelgen R, Gliemann J, Behlke J, Galatis D, et al: Molecular dissection of the interaction between amyloid precursor protein and its neuronal trafficking receptor SorLA/LR11. Biochemistry 2006;45:2618-2628.

30 Rogaeva E, Meng Y, Lee JH, Gu Y, Kawarai T, Zou F, et al: The neuronal sortilin-related receptor SORL1 is genetically associated with Alzheimer disease. Nat Genet 2007;39:168177.

31 Spoelgen R, von Arnim CA, Thomas AV, Peltan ID, Koker M, Deng A, et al: Interaction of the cytosolic domains of sorLA/LR11 with the amyloid precursor protein (APP) and beta-secretase beta-site APP-cleaving enzyme. J Neurosci 2006;26:418-428.

32 He X, Li F, Chang WP, Tang J: GGA proteins mediate the recycling pathway of memapsin 2 (BACE). J Biol Chem 2005;280:1169611703.

33 Small SA, Kent K, Pierce A, Leung C, Kang MS, Okada H, et al: Model-guided microarray implicates the retromer complex in $\mathrm{Alz}$ heimer's disease. Ann Neurol 2005;58:909919.

34 Muhammad A, Flores I, Zhang H, Yu R, Staniszewski A, Planel E, et al: Retromer deficiency observed in Alzheimer's disease causes hippocampal dysfunction, neurodegeneration, and Abeta accumulation. Proc Natl Acad Sci USA 2008;105:7327-7332.

35 Seaman MN, Marcusson EG, Cereghino JL, Emr SD: Endosome to Golgi retrieval of the vacuolar protein sorting receptor, Vps10p, requires the function of the VPS29, VPS30, and VPS35 gene products. J Cell Biol 1997; 137:79-92.

36 Seaman MN: Recycle your receptors with retromer. Trends Cell Biol 2005;15:68-75.

37 Bonifacino JS, Hurley JH: Retromer. Curr Opin Cell Biol 2008;20:427-436.

38 Attar N, Cullen PJ: The retromer complex. Adv Enzyme Regul 2010;50:216-236.

39 Hierro A, Rojas AL, Rojas R, Murthy N, Effantin $\mathrm{G}$ et al: Functional architecture of the retromer cargo-recognition complex. $\mathrm{Na}$ ture 2007;449:1063-1067.

40 Gokool S, Tattersall D, Seaman MN: EHD1 interacts with retromer to stabilize SNX1 tu- bules and facilitate endosome-to-Golgi retrieval. Traffic 2007;8:1873-1886.

41 Harterink M, Port F, Lorenowicz MJ, McGough IJ, Silhankova M, et al: A SNX3-dependent retromer pathway mediates retrograde transport of the Wnt sorting receptor Wntless and is required for Wnt secretion. Nat Cell Biol 2011;13:914-923.

42 Haft CR, de la Luz Sierra M, Bafford R, Lesniak MA, Barr VA et al: Human orthologs of yeast vacuolar protein sorting proteins Vps26, 29, and 35: assembly into multimeric complexes. Mol Biol Cell 2000;11:4105-4116.

43 Seaman MN: Cargo-selective endosomal sorting for retrieval to the Golgi requires retromer. J Cell Biol 2004;165:111-122.

44 Vieira SI, Rebelo S, Esselmann H, Wiltfang J, Lah J, et al: Retrieval of the Alzheimer's amyloid precursor protein from the endosome to the TGN is S655 phosphorylation statedependent and retromer-mediated. Mol Neurodegener 2010;5:40.

45 Lane RF, Raines SM, Steele JW, Ehrlich ME, Lah JA, et al: Diabetes-associated SorCS1 regulates Alzheimer's amyloid-beta metabolism: evidence for involvement of SorL1 and the retromer complex. J Neurosci 2010;30: 13110-13115.

46 Reitz C, Tokuhiro S, Clark LN, Conrad C, Vonsattel JP, et al: SORCS1 alters amyloid precursor protein processing and variants may increase Alzheimer's disease risk. Ann Neurol 2011;69:47-64

47 Sullivan CP, Jay AG, Stack EC, Pakaluk M, Wadlinger E, et al: Retromer disruption promotes amyloidogenic APP processing. Neurobiol Dis 2011;43:338-745.

48 Tesco G, Koh YH, Kang EL, Cameron AN, Das S, Sena-Esteves M, et al: Depletion of GGA3 stabilizes BACE and enhances betasecretase activity. Neuron 2007;54:721-737.

49 Finan GM, Okada H, Kim TW: BACE1 retrograde trafficking is uniquely regulated by the cytoplasmic domain of sortilin. J Biol Chem 2011;286:12602-12616.

50 Okada H, Zhang W, Peterhoff C, Hwang JC, Nixon RA et al: Proteomic identification of sorting nexin 6 as a negative regulator of BACE1-mediated APP processing. FASEB J 2010;24:2783-2794.

51 Bali J, Halima SB, Felmy B, Goodger Z, Zurbriggen $S$ et al: Cellular basis of Alzheimer's disease. Ann Indian Acad Neurol 2010; 13(suppl 2):S89-S93.

52 Ben Halima S, Rajendran L: Membrane anchored and lipid raft targeted $\beta$-secretase inhibitors for Alzheimer's disease therapy. J Alzheimers Dis 2011;24(suppl 2):143-152.

53 Chia PZ, Gasnereau I, Lieu ZZ, Gleeson PA: Rab9-dependent retrograde transport and endosomal sorting of the endopeptidase furin. J Cell Sci 2011;124:2401-2413.

54 Eaton S: Retromer retrieves wntless. Dev Cell 2008;14:4-6.

55 Inestrosa NC, Arenas E: Emerging roles of Wnts in the adult nervous system. Nat Rev Neurosci 2010;11:77-86. 